\title{
Hybrid Analog-Digital Precoding Design for Satellite Systems
}

\author{
Aakash Arora, Christos G. Tsinos, Bhavani Shankar Mysore R, Symeon Chatzinotas and Björn Ottersten \\ Interdisciplinary Centre for Security, Reliability and Trust (SnT), University of Luxembourg \\ email address: \{aakash.arora, christos.tsinos, bhavani.shankar, symeon.chatzinotas, bjorn.ottersten\}@uni.lu
}

\begin{abstract}
The work investigates the feasibility of massive MIMO in SatCom. Towards this, the necessary channel models, system parameters and scenarios are identified and a basic simulator developed. The work then considers an efficient implementation of the massive MIMO transmission through the use of hybrid analog/digital precoder. Efficient algorithmic solutions are proposed for the partially connected precoder architecture which enables efficiency in power/ hardware complexity and its performance evaluated.
\end{abstract}

\section{INTRODUCTION}

Multiple-Input Multiple-output (MIMO) wireless communication systems based on a Large-Scale Antenna Array (LSAA) exhibit significantly improved spectral and energy efficiency, reliability and coverage compared to the traditional ones. Furthermore, the high array gain enables them to support communication links in the millimeter-Wave (mmWave) band $(30-300 \mathrm{GHz})$ where the signal propagation is subject to severe propagation loss, penetration loss and rain fading compared to the lower frequencies. To that end, terrestrial communication systems envision them as the means to meet the exponential increasing demands for higher data rates [1]-[4]. Unfortunately, the development of transceivers based on LSAAs is a very challenging task due to high demands in hardware complexity and power consumption. A fully digital transceiver requires a dedicated Radio Frequency $(\mathrm{RF})$ chain per antenna which includes a number of different electronic elements (e.g., Digital-to-Analog/Analog-to-Digital converters) that may require prohibitive hardware complexity and power consumption as the antenna array size increases. Thus, a fully digital transceiver seems to be currently impractical and alternative approaches must be sought.

At first, analog only beamforming approaches [5]-[7] were considered as a solution to the problem. The core of these techniques is a network of analog phase shifters [8], [9], driven via a single RF chain, that imposes constant modulus constraints on the beamformer. The latter is highly desirable from a hardware complexity/power consumption perspective. Unfortunately, analog only approaches cannot support multistream communication, and thus, they usually perform poorly compared to the fully digital approaches. In an alternative approach, the phase shifters are replaced by analog switches [10]-[12] which are simpler components. However, this comes

This work is supported by the National Research Fund (FNR), Luxembourg under the AFR-PPP grant for Ph.D. project SPASAT (Ref.: 11607283), the CORE-PPP project PROSAT, ECLECTIC, and CI-PHY. at the cost of loss in array gain which again results in performance degradation, especially for highly correlated channels, like the ones experienced in the mmWave band.

The performance can be significantly improved if a hybrid Analog/Digital (A/D) transceiver architecture is employed. This approach is based on a two stage setup that consists of a low dimensional digital precoder applied in the BaseBand (BB) and an analog beamformer applied in the RF domain [13]. The RF processing part is usually implemented via a network of variable phase shifters. Two common variations of the hybrid techniques are the fully connected one, where each of the RF chains is connected to all of the available antennas and the partially connected one, where each RF chain is connected to only some of them.

In mmWave bands, hybrid transceivers were first introduced in [13], [14]. Those approaches aim at hybrid solutions that maximize the spectral efficiency of the system when a fully connected architecture is assumed. The RF precoder is designed based on a pre-determined codebook and thus, the resulting problem can be solved via the recent advances in sparse reconstruction signal processing literature [15], [16]. Codebook free hybrid transceiver designs with improved performance were also developed in [17], [18]. In [17], the techniques were developed for both the fully and the partially connected architecture and for single- and multicarrier systems that are based on the Orthogonal Frequency Division Multiplexing (OFDM) technique. Hybrid precoders for the partially connected architecture were also considered in [19]-[21].

The gains of using LSAA in terrestrial is well known and it immediately motivates their application to SatCom, about which, very little is known. The use of LSAA in SatCom would enable opportunities to increase the service quality and coverage significantly while reducing processing complexity and imparting robustness. However, adapting the terrestrial technology to SatCom is not straightforward as the two set-ups undergo different limitations when are based on LSAAs. For example, pilot contamination in not a problem on satellite systems. Massive MIMO in multicell scenarios entails difficulties in the channel estimation operation as users located in adjacent cells might inject interference into the estimation process. In the multibeam satellite case, this does not occur since the number of adjacent beams in a given area is limited. Also, the pilot signals of adjacent beams are orthogonal, and the satellite channel is, in general, non-frequency-selective 
and preserves the orthogonality at the UT. Moreover, the cochannel interference power does not decrease as the number of beams increases. Further, the favorable propagation in massive MIMO does not occur in multibeam satellite systems. That is, in a scattered terrestrial channel environment, the off-diagonal elements of the channel covariance matrix tend to zero as the number of antennas grows, leading to an ideal interferencefree scenario. On the contrary, due to the low scatter in the satellite channel, there is always strong co-channel interference among beams independent of the dimension of the multibeam satellite system. This can be construed as a manifestation of LoS (line of Sight) channels. In this context, an investigation of the LSAA applied to SatCom systems is pertinent.

Current satellites are subject to limitations on the processing power and thus, existing on-board processing for satellites with multi-antenna arrays is based mainly on a simple beamforming network that either is fixed or adaptive [22][24]. More sophisticated solutions involve the combination of the aforementioned beamforming network with digital ground based precoding that offers more flexibility. These beamforming approaches are used to create a large number of beams. In terms of utilizing the multiple beams beyond the conventional usage, initial studies in [25] suggest MIMOmultiuser applications rather than conventional point-to-point MIMO applications. However, due to the overarching fixed beam structure, the multiuser interference mitigation techniques cannot fully realize their potential and gains are limited to overlapping beam areas. This has also been cited as one of the reasons for the inability to mimic massive MIMO. However, the onset of LSAA with large number of feeds with wide coverage has rekindled the investigation on gains offered by LSAA in satellite communications, especially in the context of precoding/ beamforming.

On the other hand, on board processing based on fully digital precoding is something that has yet to be studied even for satellites that are based on antenna arrays of regular size (i.e. less than ten elements). This is due to the one RF chain per antenna requirement that could result in significant increase in the the payload complexity, mass, implementation and the energy consumption. Therefore, the exploitation of the capabilities of a LSAA for on-board processing is not an easy task and thus, efficient transmitter solutions have to be developed. The requirement on the number of RF chains, therefore, becomes the bottleneck in the implementation of such a system. Additionally, the digital processing (either on-ground in transparent or on-board in processed payload architectures) becomes complex with the increase in number of antennas due to limited resources such as fixed DC power budget, computational complexity on board the satellite. Therefore it is difficult to realize a fully-digital precoder/ beamformer. To that end, the hybrid A/D solutions seem to be a good candidate that offer a good trade-off between the performance and the transmitter's processing capabilities that could lead to viable solutions that approach the gains of a fully digital on-board precoder.

In detail, the contributions of the work reported on the present work are as follows. At first a simulator for LSAA system in SatCom is implemented incorporating appropriate channel model, system parameters and scenario. Simple transceivers, assuming large dimensional precoders with ideal channel are considered and their performance evaluated. Apart from assessing the gains, it is equally pertinent to investigate the implementation feasibility of transceiver techniques in LSAA for SatCom. A hybrid analog/digital precoder is developed for onboard processing at satellites based on LargeScale Antenna Arrays (LSAA). The proposed approach aims on the implementation of a hybrid version of the wellknown Zero-Forcing (ZF) precoding [26]. ZF is a simple and efficient way to support multi-user communication that have been extensively studied in terrestrial communications. It is also suitable for the typical satellite downlink operation. Efficient algorithmic solutions for the precoding problem are proposed for fully connected and partially connected hybrid architectures. The evaluation of the derived approaches is done via simulations over realistic channel models. As it is shown, the proposed approaches enable the use of the power and hardware complexity efficient hybrid architectures while achieving satisfactory performance. The spectral efficiency of the solutions is compared to the optimal fully digital one and to a fully analog beamforming approach, also proposed herein. For further conclusions, comparisons with respect the corresponding energy efficiency are also conducted. To that end, the power consumption of each one of the examined architectures is approximated by appropriate models.

The remainder of the paper is organized as follows. In Section 2, the system model is described. In Section 3, the problem formulation, proposed solutions and sample performance are given while Sec. 4, concludes this paper.

\section{SySTEM MODEL}

This section is divided in four sub-sections. In the first one, the system model will be described. In the second one, the proposed hybrid architectures will be described. In the third one, the metrics used to evaluate the performance of the proposed solutions will be defined. In the final section, the considered realistic channel model will be presented.

\section{A. System Description}

Let us assume the forward link of a broadband satellite system at which $K$ users are served by a gateway. It is further assumed that the satellite is equipped with a LSAA of $N>>$ $K$ elements and $M$ RF chains such that $K \leq M<<N$. The satellite is assumed to be employed with a transparent payload. The link between the gateway and the satellite is assumed to be implemented via a Frequency Division Multiplexing (FDM) strategy. That is the data for each one of the $K$ users are conveyed to the satellite via orthogonal frequency channels. Then, the received data at the satellite are transmitted to the users simultaneously by exploiting the spatial degrees of freedom that are available through the existence of multiple RF chains and antenna elements. The satellite payload prior transmitting the data to the users applies a precoding matrix $\mathbf{F}$ 
to them. Thus, under the assumption of perfect communication at the gateway-to-satellite transmission case, the input-output relationship during the satellite-users transmission phase is given for the $k$ th user by

$$
y_{k}=\mathbf{h}_{k} \mathbf{f}_{k} x_{k}+\sum_{l \neq k} \mathbf{h}_{k} \mathbf{f}_{l} x_{l}+\mathbf{z}_{k},
$$

where $y_{k}, x_{k}, \mathbf{h}_{k}=\left[h_{k 1}, \ldots, h_{k N}\right], \mathbf{f}_{k}=\left[f_{1 k}, \ldots, f_{N k}\right]^{T}$ and $z_{k}$ are the received signal, transmitted symbol, channel coefficients, precoding coefficients, and the noise sample at the $k$ th user. Note that an explicit reference to the channel model will be done in Sec 2.4. We may now write (1) in its equivalent vector form, that is

$$
\mathbf{y}=\mathbf{H F x}+\mathbf{z},
$$

where $\mathbf{y}=\left[y_{1}, \ldots, y_{K}\right]^{T}, \mathbf{x}=\left[x_{1}, \ldots, x_{K}\right]^{T}, \mathbf{H}=$ $\left[\mathbf{h}_{\mathbf{1}}, \ldots, \mathbf{h}_{\mathbf{K}}\right]$ and $\mathbf{z}=\left[z_{1}, \ldots, z_{K}\right]^{T}$. The transmission is subject to a total transmission power constraint, i.e. $\|\mathbf{F}\|_{F}^{2} \leq$ $P_{\max }$, where $\|\cdot\|_{F}^{2}$ is the Frobenius norm of a matrix.

An alternative system model that will examine due to its simplicity is the one that is equipped with a LSAA of again $N$ elements and only a single RF chain. This approach reduces the required hardware complexity to the least possible one though now it can support at any timeslot only a single user. Thus, in order to serve the $K$ available users, a Time Division Multiplexing (TDM) must be employed according to which each timeslot only a single user is served. The satellite payload can apply a simple analog beamforming operation via a beamforming vector $\mathbf{f}_{k}$ resulting in an input-output relationship given by,

$$
y_{k}=\mathbf{h}_{k} \mathbf{f}_{k} x_{k}+\mathbf{z}_{k} .
$$

Note that apart from the reduced hardware complexity, the transmissions to the users are interference free though now the satellite needs $K$ timeslots to convey the symbols to the $K$ users due to the employed TDM strategy. The latter has significant impact on the systems spectral efficiency as shown in Section 2.3.

The aim in the present work is to derive the optimal precoding/beamforming matrices for the satellite when hybrid A/D or pure analog architectures are employed by them. We start from Sec 2.2, where the considered architectures are described.

\section{B. Architectures}

In a hybrid architecture the precoding operation splits into two parts, the smaller dimensional digital precoding matrix followed by a network of analog phase-shifters. In this section, we briefly describe the two most commonly used types of hybrid architectures namely, the fully-connected and the partially-connected architecture [27]. In both cases, the input signals are processed with a lower dimensional digital baseband precoder. The only change between the two configurations can be seen from the analog precoding stage. In the fully-connected case, output of each RF chain is connected to all the tranmsitting antennas as shown in Figure 1, thus

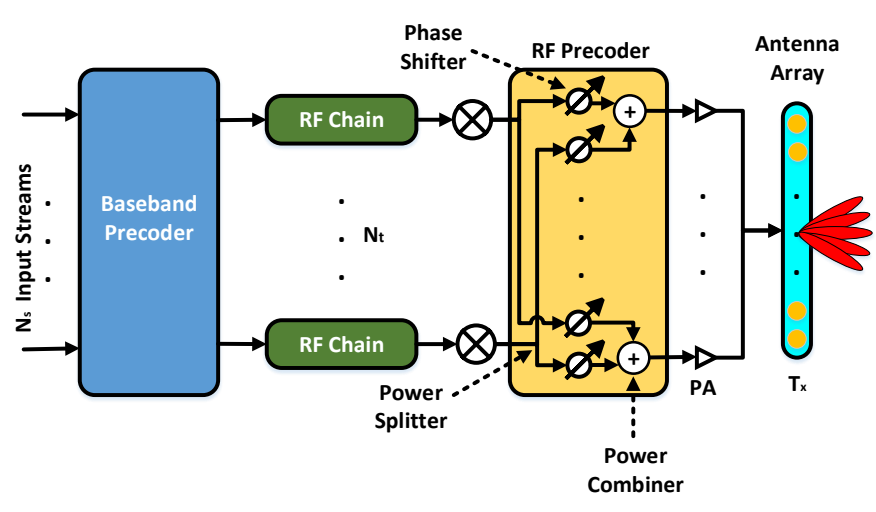

Fig. 1. Block diagram of fully-connected hybrid architecture.

each analog precoder output is a linear combination of all the RF signals. In the partially-connected case, each RF chain is connected only to a subset of transimitting antennas as shown in Figure 2. This architecture has significantly reduced

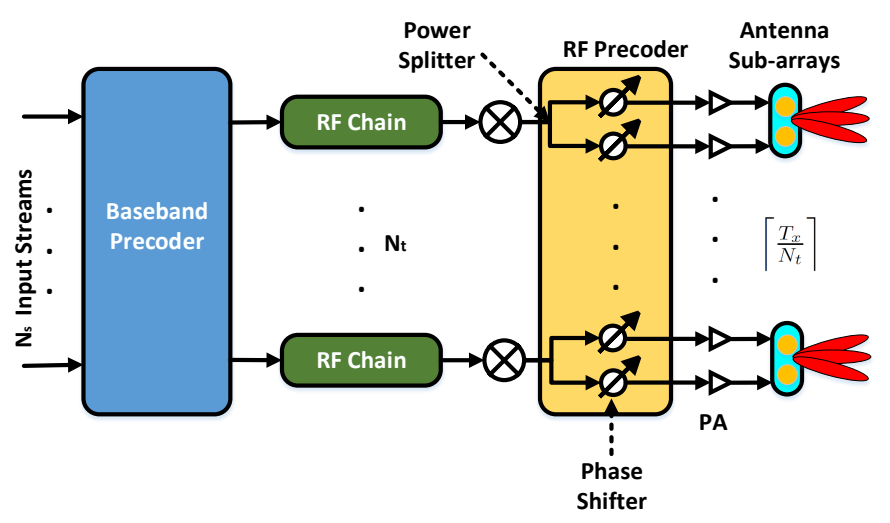

Fig. 2. Block diagram of partially-connected hybrid architecture.

complexity, but somewhat reduced performance in comparison with the fully-connected one. In the fully-connected case, the input signals are first processed with a lower dimensional digital baseband precoder followed by a network of phaseshifter chains. For hardware simplicity we focus on partiallyconnected architecture.

\section{Performance Metrics}

Here, we briefly define two metrics to study the performance of different hybrid architectures.

1) Spectral Efficiency: The spectral efficiency (SE) for a multi-user multiple-input single-output (MU-MISO) system is,

$$
\mathrm{SE}=\sum_{k=1}^{K} \log _{2}\left(1+\frac{\left|\mathbf{h}_{k}^{H} \mathbf{f}_{k}\right|^{2}}{\sum_{j \neq k}\left|\mathbf{h}_{k}^{H} \mathbf{f}_{j}\right|^{2}+\sigma_{n}^{2}}\right),
$$

where $\sigma_{n}^{2}$ is the noise variance, here $\mathbf{f}_{k}$ denotes the precoding vector from the fully-digital precoding matrix $\mathbf{F}=$ $\left[\mathbf{f}_{1}, \mathbf{f}_{2}, \ldots, \mathbf{f}_{K}\right] \in \mathbb{C}^{N \times K}$. For a hybrid architecture the SE expression can be derived by replacing $\mathbf{F}$ with $\mathbf{F}_{\mathrm{RF}} \mathbf{F}_{\mathrm{BB}}$ where $\mathbf{F}_{\mathrm{BB}} \in \mathbb{C}^{M \times K}$ is the digital baseband precdoing matrix 
and $\mathbf{F}_{\mathrm{RF}}$ is the analog precoding matrix with dimensions $N \times M$. For a fully-connected architecture, each entry of ana$\log$ precoding matrix is unit-modulus, whereas for a partiallyconnected architecture it has the following structure,

$$
\mathbf{F}_{\mathrm{RF}}=\left[\begin{array}{cccc}
\tilde{\mathbf{f}}_{\mathrm{RF}_{1}} & \mathbf{0} & \ldots & \mathbf{0} \\
\mathbf{0} & \tilde{\mathbf{f}}_{\mathrm{RF}_{2}} & \ldots & \mathbf{0} \\
\vdots & & \ddots & \mathbf{0} \\
\mathbf{0} & \mathbf{0} & \ldots & \tilde{\mathbf{f}}_{\mathrm{RF}_{K}}
\end{array}\right]
$$

where $\tilde{\mathbf{f}}_{\mathrm{RF}_{i}}$ is a vector of dimensions $\frac{N}{M} \times 1$ having unitmodulus entries. Therefore, we have the following expression,

$$
\mathrm{SE}=\sum_{k=1}^{K} \log _{2}\left(1+\frac{\left|\mathbf{h}_{k}^{H} \mathbf{F}_{\mathrm{RF}} \mathbf{f}_{\mathrm{BB}_{k}}\right|^{2}}{\sum_{j \neq k}\left|\mathbf{h}_{k}^{H} \mathbf{F}_{\mathrm{RF}} \mathbf{f}_{\mathrm{BB}_{j}}\right|^{2}+\sigma_{n}^{2}}\right),
$$

where $\mathbf{F}_{\mathrm{BB}}=\left[\mathbf{f}_{\mathrm{BB}_{1}}, \mathbf{f}_{\mathrm{BB}_{2}}, \ldots, \mathbf{f}_{\mathrm{BB}_{K}}\right]$. For an analog system, the $\mathrm{SE}$ can be written as,

$$
\mathrm{SE}=\sum_{k=1}^{K} \log _{2}\left(1+K^{-1}\left|\mathbf{h}_{k}^{H} \mathbf{f}_{k}\right|^{2}\right),
$$

where $\mathbf{f}_{k}$ represents the analog beamforming vector for $k$-th user.

2) Energy Efficiency: Apart from SE another useful metric for performance analysis is the energy efficiency (EE) [19], [28]-[32]. It can be defined as the ratio of the achieved SE to the consumed power,

$$
\mathrm{E}(\mathbf{F})=\frac{\mathrm{SE}}{\mathrm{P}(\mathbf{F})},
$$

where $\mathrm{P}(\mathbf{F})$ is the consumed power. Now, we analyze the power consumption in the fully-digital and the different hybrid architectures.

It is evident that the power consumption of the different architectures is required for evaluating their energy efficiency. An exact computation of the dissipated power is a very difficult task and thus, our analysis is based on approximate power consumption models which are commonly adopted in relevant literature works [29], [33].

Let us start with the case of a fully digital transmitter. Based on the analysis given in [33], its power consumption $P_{D}(\mathbf{F})$ can be approximated by

$$
P_{D}(\mathbf{F}) \approx\|\mathbf{F}\|_{F}^{2} / \eta+N P_{R F}^{T}+P_{L O},
$$

where $\eta$ is the efficiency of the Power Amplifiers (PA), $P_{R F}^{T}=$ $P_{D A C}+P_{m i x}+P_{f i l}$ and $P_{R F}^{R}=P_{A D C}+P_{m i x}+P_{f i l}$ is the power consumed in a transmitter's RF chain, respectively, $P_{D A C}, P_{m i x}, P_{f i l}$, is the power consumed by the Digital-toAnalog Converter (DAC), the mixer and filters, respectively.

For the hybrid transmitters, the power consumption on the electronic components of the RF chains is significantly reduced due to the in general reduced number of the latter. However, there is power consumed for the operation of the phase shifting network. Thus, by following the analysis in [28], it can be shown that the power consumption of the fully connected $P_{F C H}$ and the partially connected $P_{P C H}$ architectures, is respectively approximated by

$$
\begin{aligned}
& P_{F C H}\left(\mathbf{F}_{R F}, \mathbf{F}_{B B}\right) \approx\left\|\mathbf{F}_{R F} \mathbf{F}_{B B}\right\|_{F}^{2} / \eta \\
& +M P_{R F}^{T}+P_{L O}+N M P_{P s}, \\
& P_{P C H}\left(\mathbf{F}_{R F}, \mathbf{F}_{B B}\right) \approx\left\|\mathbf{F}_{R F} \mathbf{F}_{B B}\right\|_{F}^{2} / \eta \\
& +M P_{R F}^{T}+P_{L O}+N P_{P s} .
\end{aligned}
$$

The power consumption reduction due to the employment of a partially connected architecture over a fully connected one can be seen by comparing (10) to (11). This reduction mainly stems from the fact that the first one requires only $N$ phase shifters while the second one requires $N M$.

From a first comparison of (9)-(11), one may see that there is a potential to reduce significantly the power consumption of a transceiver by resorting to a hybrid solution, at least when the latter is based on a relative small number of RF chains.

\section{Channel}

A narrowband clustered channel is considered based on the Saleh-Valenzuela model is typically used to represent the mmWave channel and it captures the effects including severe path loss, limited scattering, high correlation among antennas due to closely packed antenna arrays, etc. The channel model from the antenna array to the $k$ th user to takes the form,

$$
\mathbf{h}_{k}=\sqrt{\frac{1}{L}} \sum_{l=1}^{L} \alpha_{k, l} \mathbf{a}_{t}^{T}\left(\phi_{k, l}, \theta_{k, l}\right)
$$

where $L$ is the number of paths, $\mathbf{a}_{t}\left(\Phi_{k, l}, \theta_{k, l}\right)$ denotes the $N \times 1$ steering vector for the $k$ th user on the $l$ th path and $\alpha_{k, l}$ denotes the corresponding path attenuation.

1) Antenna Pattern: In this study, the antenna pattern per takes the form,

$$
G(\Phi, \theta)=10^{\frac{-3}{20}\left(\frac{\theta}{\theta_{3 d B}}\right)^{2}} \times 10^{\frac{-3}{20}\left(\frac{\phi}{\theta_{3 d B}}\right)^{2}}
$$

where, $\theta_{3 d B}=\frac{33 \lambda}{d}, \lambda$ is the wavelength and $d$ is the interelement spacing. Note that a separable model in the two dimensions, $\theta, \phi$.

2) LOS Channel: Due to the separability of the antenna pattern in (13) and lack of scatterers on the downlink, the channel model in (12) can be simplified as,

$$
\begin{aligned}
\mathbf{h}_{k} & =\alpha_{k}\left(\mathbf{a}_{t}^{T}\left(\theta_{k}\right) \otimes \mathbf{a}_{t}^{T}\left(\phi_{k}\right)\right), \quad \text { where } \\
\mathbf{a}_{t}^{T}\left(\theta_{k}\right)(m) & =e^{-j 2(m-1) \pi \frac{d \sin \left(\theta_{k}\right)}{\lambda}}, m \in[1, N] .
\end{aligned}
$$

\section{Problem Formulation, Solutions And SAMPle PERFORMANCE}

In order to design a precoder based on hybrid-architecture, we provide a two-step approach. At first, the fully-digital precoder is obtained using conventional precoding techniques like ZF, minimum mean squared error (MMSE), etc. We propose to choose $\mathrm{ZF}$ as the fully-digital precoder. For the system model presented in (2), the ZF precoder is given by,

$$
\mathbf{F}=\mathbf{H}^{H}\left(\mathbf{H H}^{H}\right)^{-1} \text {. }
$$


The second step is to obtain the hybrid precoders from the fully-digital precoder. Similar to the point-to-point MIMO case, we design optimal hybrid precoders by minimizing the Frobenius norm of the difference between the fully-digital and the hybrid precoders. Therefore, we formulate the following problem to design hybrid precoders,

$$
\begin{gathered}
\min _{\mathbf{F}_{\mathrm{RF}}, \mathbf{F}_{\mathrm{BB}}}\left\|\mathbf{F}-\mathbf{F}_{\mathrm{RF}} \mathbf{F}_{\mathrm{BB}}\right\|_{F}^{2} \\
\text { subject to }\left\|\mathbf{F}_{\mathrm{RF}} \mathbf{F}_{\mathrm{BB}}\right\|_{F}^{2}=K \\
\mathbf{F}_{\mathrm{RF}} \in \mathcal{A},
\end{gathered}
$$

where $\mathcal{A}$ denotes the constraint set imposing unit-modulus and structural constraints on the analog precoder $\mathbf{F}_{\mathrm{RF}}$. In fullyconnected case, the set $\mathcal{A}=\left\{\left.\mathbf{F}_{\mathrm{RF}}|| \mathbf{F}_{\mathrm{RF}}(i, j)\right|^{2}=1, \forall i, j\right\}$, whereas for the partially-connected case the set $\mathcal{A}$ describes the analog precoding structure as shown in (5), with each nonzero vector having the unit-modulus entries.

It is straightforward to see that the problem (17) is a nonconvex problem because both the objective as well as the constraints are nonconvex. In literature, there exists different algorithms to solve the problem for different hybrid architectures [17]. But these algorithms are not computationally efficient and therefore, their applicability for large-scale antenna array systems is an issue. Given the limited capabilities of the satellite payload, the computational demanding existing approaches cannot be applied for onboard processing. Thus, such techniques can only be applied offline on the gateway, thus this introduces additional overhead fro the feedback of the precoder coefficients from the gateway to the satellite. In addition such a strategy adds additional latencies that may have impact on the system's performance when applying to fast varying environments. To that end, we developed computational efficient methods for the solution of the optimization problem in (17) that enable onboard computation of the precoder coefficients. In order to alleviate these issues, we have a recent submitted work, which developed efficient algorithms to solve this problem for different hybrid architectures.

\section{A. Sample Performance}

We now present a sample numerical simulation result to evaluate the performance of different hybrid architecture in comparison with the fully-digital precoder. The simulation scenario we have considered is as follows, $N=700, M=10$ and $K=10$. It should be noted that a global coverage is considered involving $\theta, \in[-9,9]^{\circ}$ and $\phi \in[-10,10]^{\circ}$. The user terminals are chosen at random in this region ${ }^{1}$. The fully digital precoder provides performance of about $10 \mathrm{bps} / \mathrm{Hz}$ when all the 700 elements are used at $10 \mathrm{~dB}$ SNR. The of the lowcomplexity partially connected architecture does not perform well due to inherent shortcomings in the architecture.

\footnotetext{
${ }^{1}$ It should be noted that the performance is determined by the SINR while the plot is wrt SNR (or $\mathrm{C} / \mathrm{N}$ ) since the user locations vary and so does the SIR (Signal-to-Interference-Ratio). The SINR is then obtained $S I N R=\frac{1}{\frac{1}{S N R}+\frac{1}{S I R}}$.
}

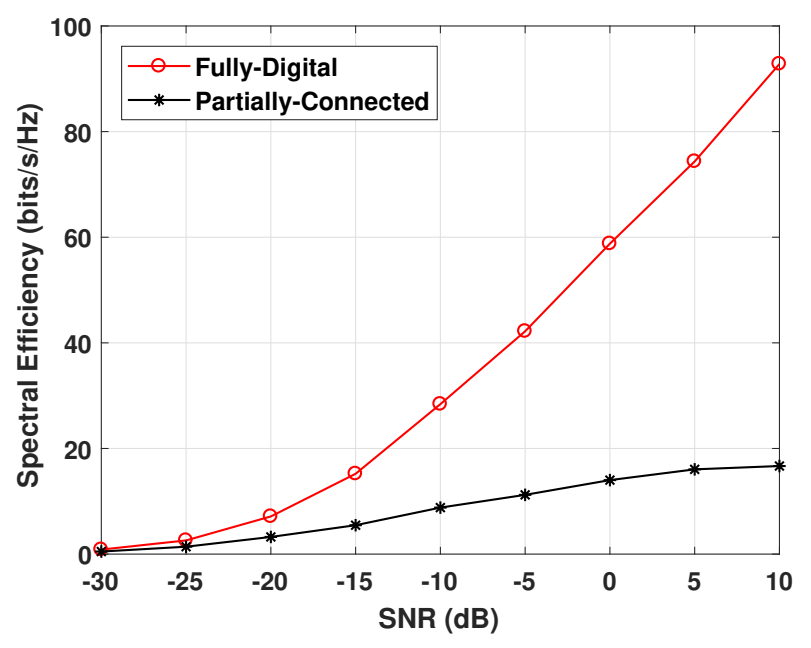

Fig. 3. Spectral efficiency comparison versus SNR.

\section{CONCLUSION}

This work presents a first investigation on the use of a hybrid analog/digital precoder is developed for onboard processing at satellites based on Large-Scale Antenna Arrays (LSAA). Solutions are proposed for fully connected and partially connected hybrid architectures and a sample evaluation of their performance is evaluated. Future studies include further investigation of partially and fully connected architectures for different dimensions and users. Comparison of the proposed method with an existing system with a given beam structure and a study of the impact of imperfect channel estimation on the techniques and the development of robust counterparts.

\section{REFERENCES}

[1] T. Bai and R. W. Heath, "Coverage and rate analysis for millimeter-wave cellular networks," IEEE Trans. on Wireless Communications, vol. 14, no. 2, pp. 1100-1114, Feb 2015.

[2] T. S. Rappaport, S. Sun, R. Mayzus, H. Zhao, Y. Azar, K. Wang, G. N. Wong, J. K. Schulz, M. Samimi, and F. Gutierrez, "Millimeter wave mobile communications for 5G cellular: It will work!" IEEE Access, vol. 1, pp. 335-349, 2013.

[3] S. K. Yong and C.-C. Chong, "An overview of multigigabit wireless through millimeter wave technology: potentials and technical challenges," EURASIP Journal on Wireless Communications and Networking, vol. 2007, no. 1, pp. 1-10, 2006.

[4] Z. Pi and F. Khan, "An introduction to millimeter-wave mobile broadband systems," IEEE Communications Magazine, vol. 49, no. 6, pp. 101-107, June 2011.

[5] S. Hur, T. Kim, D. J. Love, J. V. Krogmeier, T. A. Thomas, and A. Ghosh, "Millimeter wave beamforming for wireless backhaul and access in small cell networks," IEEE Trans. on Communications, vol. 61, no. 10, pp. 4391-4403, Oct. 2013.

[6] T. Baykas, C. S. Sum, Z. Lan, J. Wang, M. A. Rahman, H. Harada, and S. Kato, "IEEE 802.15.3c: the first IEEE wireless standard for data rates over $1 \mathrm{~Gb} / \mathrm{s}$," IEEE Communications Magazine, vol. 49, no. 7, pp. 114-121, July 2011.

[7] D. J. Love and R. W. Heath, "Equal gain transmission in multiple-input multiple-output wireless systems," IEEE Trans. on Communications, vol. 51, no. 7, pp. 1102-1110, July 2003.

[8] C. H. Doan, S. Emami, D. A. Sobel, A. M. Niknejad, and R. W. Brodersen, "Design considerations for $60 \mathrm{GHz}$ CMOS radios," IEEE Communications Magazine, vol. 42, no. 12, pp. 132-140, Dec 2004. 
[9] A. Hajimiri, H. Hashemi, A. Natarajan, X. Guan, and A. Komijani, "Integrated phased array systems in silicon," Proceedings of the IEEE, vol. 93, no. 9, pp. 1637-1655, Sept 2005.

[10] A. F. Molisch, M. Z. Win, Y.-S. Choi, and J. H. Winters, "Capacity of MIMO systems with antenna selection," IEEE Trans. on Wireless Communications, vol. 4, no. 4, pp. 1759-1772, July 2005.

[11] A. Gorokhov, D. A. Gore, and A. J. Paulraj, "Receive antenna selection for MIMO spatial multiplexing: theory and algorithms," IEEE Trans. on Signal Processing, vol. 51, no. 11, pp. 2796-2807, Nov 2003.

[12] S. Sanayei and A. Nosratinia, "Antenna selection in mimo systems," IEEE Communications Magazine, vol. 42, no. 10, pp. 68-73, Oct 2004

[13] O. E. Ayach, S. Rajagopal, S. Abu-Surra, Z. Pi, and R. W. Heath, "Spatially sparse precoding in millimeter wave MIMO systems," IEEE Trans. on Wireless Communications, vol. 13, no. 3, pp. 1499-1513, March 2014.

[14] A. Alkhateeb, O. E. Ayach, G. Leus, and R. W. Heath, "Channel estimation and hybrid precoding for millimeter wave cellular systems," IEEE Journal of Selected Topics in Signal Processing, vol. 8, no. 5, pp. 831-846, Oct 2014.

[15] J. A. Tropp and A. C. Gilbert, "Signal recovery from random measurements via orthogonal matching pursuit," IEEE Trans. on Information Theory, vol. 53, no. 12, pp. 4655-4666, Dec 2007.

[16] J. A. Tropp, "Greed is good: algorithmic results for sparse approximation," IEEE Trans. on Information Theory, vol. 50, no. 10, pp. 22312242 , Oct 2004.

[17] X. Yu, J. C. Shen, J. Zhang, and K. B. Letaief, "Alternating minimization algorithms for hybrid precoding in millimeter wave MIMO systems," IEEE Journal of Selected Topics in Signal Processing, vol. 10, no. 3, pp. 485-500, April 2016.

[18] F. Sohrabi and W. Yu, "Hybrid digital and analog beamforming design for large-scale antenna arrays," IEEE Journal of Selected Topics in Signal Processing, vol. 10, no. 3, pp. 501-513, April 2016.

[19] X. Gao, L. Dai, S. Han, C. L. I, and R. W. Heath, "Energy-efficient hybrid analog and digital precoding for mmwave MIMO systems with large antenna arrays," IEEE Journal on Selected Areas in Communications, vol. 34, no. 4, pp. 998-1009, April 2016.

[20] J. Singh and S. Ramakrishna, "On the feasibility of codebook-based beamforming in millimeter wave systems with multiple antenna arrays," IEEE Trans. on Wireless Communications, vol. 14, no. 5, pp. 2670-2683, May 2015.

[21] J. A. Zhang, X. Huang, V. Dyadyuk, and Y. J. Guo, "Massive hybrid antenna array for millimeter-wave cellular communications," IEEE Wireless Communications, vol. 22, no. 1, pp. 79-87, February 2015.

[22] P. Angeletti and N. Alagha, "Space/ground beamforming techniques for emerging hybrid satellite terrestrial networks," 2009.

[23] N. Song, T. Yang, and M. Haardt, "Efficient hybrid space-ground precoding techniques for multi-beam satellite systems," in IEEE International Conf. Acoustics, Speech and Signal Process. (ICASSP), March 2017, pp. 6284-6288.

[24] B. Devillers, A. Perez-Neira, and C. Mosquera, "Joint linear precoding and beamforming for the forward link of multi-beam broadband satellite systems," in IEEE Global Telecommun. Conf. - GLOBECOM 2011, Dec 2011, pp. 1-6.

[25] P. Arapoglou, K. Liolis, M. Bertinelli, A. Panagopoulos, P. Cottis, and R. De Gaudenzi, "MIMO over Satellite: A Review," IEEE Communications Surveys Tutorials, vol. 13, no. 1, pp. 27-51, First 2011.

[26] M. Ghosh, "A comparison of normalizations for ZF precoded MUMIMO systems in multipath fading channels," IEEE Wireless Commun. Lett., vol. 2, no. 5, pp. 515-518, October 2013.

[27] A. F. Molisch, V. V. Ratnam, S. Han, Z. Li, S. L. H. Nguyen, L. Li, and K. Haneda, "Hybrid Beamforming for Massive MIMO: A Survey," IEEE Communications Magazine, vol. 55, no. 9, pp. 134-141, Sep. 2017.

[28] C. G. Tsinos, S. Maleki, S. Chatzinotas, and B. Ottersten, "On the energy-efficiency of hybrid analogdigital transceivers for single- and multi-carrier large antenna array systems," IEEE Journal on Selected Areas in Communications, vol. 35, no. 9, pp. 1980-1995, Sep. 2017.

[29] R. Mendez-Rial, C. Rusu, N. Gonzlez-Prelcic, A. Alkhateeb, and R. W. Heath, "Hybrid MIMO architectures for millimeter wave communications: Phase shifters or switches?" IEEE Access, vol. 4, pp. 247-267, 2016.

[30] A. J. G. Rodriguez, V. Venkateswaran, P. Rulikowski, and C. Masouros, "Hybrid analog-digital precoding revisited under realistic RF modeling," IEEE Wireless Communications Letters, vol. PP, no. 99, pp. 1-1, 2016.
[31] P. Sudarshan, N. B. Mehta, A. F. Molisch, and J. Zhang, "Antenna selection with RF pre-processing: robustness to RF and selection nonidealities," in Radio and Wireless Conference, 2004 IEEE, Sept 2004, pp. 391-394.

[32] V. Venkateswaran, F. Pivit, and L. Guan, "Hybrid RF and digital beamformer for cellular networks: Algorithms, microwave architectures, and measurements," IEEE Trans. on Microwave Theory and Techniques, vol. 64, pp. 2226-2243, July 2016.

[33] S. Cui, A. Goldsmith, and A. Bahai, "Energy-efficiency of MIMO and cooperative MIMO techniques in sensor networks," IEEE Journal on Selected Areas in Communications, vol. 22, no. 6, pp. 1089-1098, Aug 2004. 